Cahiers de civilisation médiévale

\title{
Collectif, Polity and Neighbourhood in Early Medieval Europe
}

\section{Corinne Péneau}

\section{OpenEdition}

1 Journals

Édition électronique

URL : https://journals.openedition.org/ccm/8107

DOI : $10.4000 / \mathrm{ccm} .8107$

ISSN : 2119-1026

Éditeur

Centre d'études supérieures de civilisation médiévale/Université de Poitiers

\section{Édition imprimée}

Date de publication : 1 septembre 2021

Pagination : 278-281

ISBN : 978-2-490783-10-6

ISSN : 0007-9731

\section{Référence électronique}

Corinne Péneau, "Collectif, Polity and Neighbourhood in Early Medieval Europe », Cahiers de civilisation médiévale [En ligne], 255 | 2021, mis en ligne le 01 septembre 2021, consulté le 04 février 2022. URL : http://journals.openedition.org/ccm/8107; DOl : https://doi.org/10.4000/ccm.8107

\section{(c) $(7)$}

La revue Cahiers de civilisation médiévale est mise à disposition selon les termes de la Licence Creative Commons Attribution - Pas d'Utilisation Commerciale - Pas de Modification 4.0 International. 
Polity and Neighbourhood in Early Medieval Europe, Julio Escalona, Orri Vésteinsson et Stuart Brookes (dir.), Turnhout, Brepols (The Medieval Countryside, 21), 2019, DOI : 10.1484/M.TMC-EB.5.116089.

C'est à un jeu d'échelle, dont le véritable sujet est la relation entre les pouvoirs locaux et le pouvoir central, que convie cet ouvrage collectif. Il est le troisième volume issu d'une série de quatre séminaires, principalement financés entre 2011 et 2014 par le gouvernement espagnol, et organisés en Islande, en Norvège et en Espagne par un groupe de chercheurs internationaux (le « FESE2 Project Group »).
Les contributions, consacrées à la péninsule Ibérique, à l'Italie, à la Scandinavie et à l'Islande ainsi qu'à l'Angleterre anglo-saxonne, offrent des approches variées d'une problématique centrée sur les influences que le pouvoir central exerçait sur les communautés locales et sur les influences exercées par ces communautés sur les pouvoirs centraux ou intermédiaires. Elles s'appuient aussi bien sur les sources écrites que sur les apports de l'archéologie ou de la toponymie. 
Les exemples développés sont souvent très précis, illustrés par des schémas et de nombreuses cartes, mais le non-spécialiste pourra regretter des mises en contexte très succinctes et parfois trop allusives, dans des articles qui privilégient généralement les synthèses historiographiques et la mise à l'épreuve de grands modèles théoriques.

Contrairement à ce qu'affirme le titre, le Moyen Âge central, en particulier dans le contexte scandinave, est également pris en compte. Les études peuvent être consacrées aux traces laissées par les États disparus sur les communautés locales. Un bel exemple est donné par Letty Ten Harkel dans son étude consacrée à la forteresse circulaire de l'île de Walcheren (actuels Pays-Bas), construite à l'époque viking, qui fut réutilisée par la suite dans un contexte régional. Plusieurs contributions illustrent le besoin d'avoir symboliquement recours à un pouvoir central, fût-il fantomatique. Wendy Davies identifie ainsi des micro-régions à partir des chartes du nord de la péninsule Ibérique qui utilisent, longtemps après la disparition du royaume wisigothique, des formules qui étaient en usage dans ses chartes. Le volume explore le jeu des influences croisées entre pouvoirs locaux et centraux, même si la majorité des articles est principalement consacrée aux effets de l'exercice du pouvoir central sur les communautés locales, comme sa capacité à créer des divisions administratives - ce qui est particulièrement mis en valeur dans les articles concernant l'Angleterre anglo-saxonne - ou à imposer de nouvelles habitudes, par exemple de nouveaux modes de sépulture ou de nouvelles conceptions de la propriété.

La formation même des États, définis ici, sans tenir toujours compte de l'efficacité de leurs compétences administratives, comme des pouvoirs centraux et supra-régionaux, occupe aussi une place de choix dans le volume. Les pouvoirs centraux sont-ils le résultat d'une construction progressive, liée à l'agglutination de pouvoirs locaux, ou sont-ils au contraire la force centrifuge qui conduit à la formation de grandes entités territoriales? La réponse ne saurait être unique et les différents cas envisagés soulignent « the bidirectional nature of the relationship between localities and larger-scale social processes », selon la formule de Julio Escalona (p. 351).

La question est, par exemple, examinée à travers le degré de connaissance que les élites locales possédaient des pouvoirs extérieurs et la manière dont ils réinvestissaient localement ce savoir. La connaissance des territoires était, à une époque où les cadastres avaient disparu en Occident, un élément-clef des relations entre les élites locales et les pouvoirs centraux. J. Escalona forge ainsi la notion de Dense Local Knowledge pour désigner ce capital symbolique, qui peut servir d'interface entre les pouvoirs locaux et les pouvoirs plus lointains. Étudiant les changements de propriétés dans un ensemble de chartes du monastère de Cardeña en Castille au $\mathrm{x}^{\mathrm{e}} \mathrm{s}$., il montre que les élites s'appuyaient sur les connaissances territoriales fines des habitants, mobilisés en particulier en tant que témoins, pour bâtir et gérer leur patrimoine. Iñaki Martín Viso montre, en revanche, que l'extension du royaume astur-léonais au sud du Duro s'est faite selon des principes opposés, les nouvelles élites imposant de nouvelles normes territoriales, en particulier de nouveaux cimetières, sans tenir aucun compte des anciens lieux de sépulture. Dans l'Angleterre anglo-saxonne, le Domesday Book a imposé un nouveau maillage administratif, contrôlé par le pouvoir royal, mais Stuart Brookes et Andrew Reynolds formulent des hypothèses à partir de l'archéologie et de la toponymie pour expliquer l'émergence de territoires formés autour d'anciennes communautés bien structurées, mais aussi par l'exclusion hors de ce système, par la formation d'unités spécifiques, par exemple des territoires marginaux ou peuplés de minorités comme des Danois installés dans l'ancien Danelaw. L'exclusion apparente de ces populations et de ces espaces du système ordinaire n'était, en réalité, qu'une stratégie pour mieux les intégrer à l'administration royale.

Particulièrement remarquable est la synthèse d'Orri Vésteinsson sur l'Islande (« State Formation and World View: Commonwealth Island as a Part of the Norvegian Realm »). Cette contribution offre une interprétation globale très convaincante des relations entre l'Islande et la Norvège du $\mathrm{x}^{\mathrm{e}} \mathrm{s}$. à 1262 , année où l'île passa sous la domination du roi de Norvège. Dans l'historiographie classique, cette période est celle du " miracle islandais », dont les sagas ont largement contribué à forger la mythologie. La petite île indépendante a réussi à vivre sans roi pendant plusieurs siècles, à construire, grâce à ses lois, une sorte de république relativement stable entre les $\mathrm{XI}^{\mathrm{e}}$ et $\mathrm{XII}^{\mathrm{e}} \mathrm{S}$., mais, déchirée par des guerres civiles à partir de 1220 , l'île connut des troubles qui conduisirent à sa domination par le pouvoir norvégien. Une vision plus récente est celle de Jón Viðar Sigurðsson : les chefs 
islandais, les goðar, qui imposaient leur domination sur de petits groupes d'hommes, commencèrent, dès $l e \mathrm{XI}^{\mathrm{e}} \mathrm{s}$., à perdre leur influence au profit de cinq grandes familles qui en vinrent à dominer l'île au $\mathrm{XIII}^{\mathrm{e}} \mathrm{s}$. L'Église islandaise, de plus en plus soumise à l'archevêché de Nidaros, participait aux changements politiques sur l'île. Toutefois, cette vision renouvelée s'intéresse au seul territoire islandais; $O$. Vésteinsson propose de briser cette logique encore très nationale, pour ne pas dire nationaliste, en prenant en compte la forte implication des élites islandaises, y compris des auteurs islandais des sagas, dont beaucoup furent rédigées pour les rois de Norvège, dans la vie politique de ce royaume. Ces élites trouvaient dans leurs séjours en Norvège, dans leur fréquentation de la cour, un prestige qui pouvait rejaillir sur toute leur famille, ce que les sagas mettaient bien en relief à travers le récit d'Islandais qui, bien que mal adaptés à la vie de cour, avaient réussi par leurs talents, militaires ou littéraires, à gagner la confiance, voire l'amitié du roi. Un cadeau royal (un bateau ou une épée) apportait la preuve de cette relation privilégiée et garantissait à son propriétaire, de retour en Islande, un prestige inégalé. Loin d'être un pouvoir lointain, la monarchie norvégienne était, bien avant 1262, une force structurante dans la vie politique islandaise. Le capital politique obtenu grâce à la fréquentation, par un individu, de la cour norvégienne était réinvesti en Islande et profitait à toute sa famille. Ainsi, le système judiciaire et législatif acéphale créé par les Islandais dès le $\mathrm{x}^{\mathrm{e}} \mathrm{s}$. ne peut être vu comme un système républicain, car les Islandais reconnaissaient déjà implicitement « une source extérieure d'autorité suprême » (p. 220). La conception que les élites islandaises se faisaient du pouvoir dépassait donc largement les limites de leur propre territoire. L'intérêt de ces élites à fréquenter le Royaume de Norvège facilita l'implantation d'un pouvoir de nature étatique en Islande.

Cette adhésion des élites islandaises à l'État norvégien, même encore embryonnaire, pose toutefois la question concomitante des conditions d'émergence d'un pouvoir centralisé en Norvège. La figure, presque mythique, du roi Harald hárfagri ( « aux Beaux Cheveux ») ne permet pas de dater la formation du Royaume de Norvège, mais il n'en demeure pas moins vrai qu'émergea, au cours du IX ${ }^{\mathrm{e}} \mathrm{s}$., une nouvelle organisation politique, bien visible à travers l'abandon des courtyard sites au profit de neuf thing principaux. Ces courtyard sites sont étudiés dans un article de Frode Iversen (« The Thing and the King: the Formation of the Norwegian Medieval Kingdom »). Apparues dès la fin de l'Antiquité, parfois dès le $\mathrm{II}^{\mathrm{e}} \mathrm{S}$., ces cours ovales ou semi-circulaires entourées de halles en bois ont eu différentes fonctions, commerciales ou militaires. Elles ont sans doute aussi joué un rôle politique en étant des lieux de réunion pour les habitants d'une région définie. Le premier de ces sites archéologiques a été découvert dans les années 1860. Aujourd'hui, une trentaine de sites sont connus le long de la côte norvégienne. Certains étaient encore en usage au $\mathrm{X}^{\mathrm{e}} \mathrm{s}$. et le restèrent jusqu'au $\mathrm{XI}^{\mathrm{e}} \mathrm{s}$. F. Iversen, spécialiste de l'archéologie des thing et autres lieux de rassemblements politiques, étudie la reconfiguration de ces lieux de réunion et son rôle dans l'émergence, entre le $\mathrm{IX}^{\mathrm{e}}$ et le $\mathrm{x}^{\mathrm{e}} \mathrm{s}$., $\mathrm{du}$ Gulatinglag, une vaste région du sud-ouest de la Norvège, dont les lois étaient créées par l'assemblée (ou thing) de Gulen. Ces régions dépendantes d'un thing ayant le pouvoir de créer ou de modifier la loi étaient une vingtaine en Scandinavie au Moyen Âge central. Le terme de thing peut, en effet, désigner plusieurs types d'assemblée : en vieux norvégien, plus de vingt-cinq termes différents peuvent les nommer, selon le moment où elles se tenaient dans l'année, selon la cause traitée ou selon la région touchée par ce rassemblement, local ou plus large, au niveau d'un district, de la moitié ou du tiers de ce district ou pour une province tout entière. Le thing avait des rôles judiciaire et législatif. Il n'existait pas de droit d'appel d'un thing à un autre et la hiérarchie entre ces assemblées était donc toute relative. Les thing au niveau des principales provinces rassemblaient les plus importants des propriétaires, qui avaient un rôle représentatif, alors que les assemblées locales étaient ouvertes à tous. Entre le $\mathrm{XI}^{\mathrm{e}}$ et la fin du XIII ${ }^{\mathrm{e}} \mathrm{s}$., le nombre de ces délégués baissa de 345 (ou plus) à 148 au Gulating. Au XIII ${ }^{\mathrm{e}}$ s., l'influence du pouvoir royal était déjà forte et le lagman, qui présidait le thing, était alors nommé par le roi. À cette époque, les différents districts, où se tenaient les assemblées, avaient perdu leur vocation militaire : la levée armée (ou leiðrang) avait été transformée en impôt. La réduction du nombre des délégués permettait sans doute au roi de mieux contrôler l'assemblée, mais elle peut aussi être liée à des mesures d'économie, car ces délégués recevaient chacun une compensation financière, En 1274, la rédaction, à la demande du roi Magnus Lagabøter, d'une loi valable dans tout le 
Royaume de Norvège constitue l'étape ultime dans le processus d'unification des lois. Au cours du XIV s., sept des neuf thing principaux furent délocalisés dans les villes proches.

Si la fin du processus est connue, ses débuts, en particulier la création même des thing provinciaux, l'est beaucoup moins et c'est ce que F. Iversen souhaite mettre en lumière à travers une hypothèse stimulante : l'archéologie des courtyard sites pourrait aussi être une archéologie des thing, en mettant à jour le processus de réorganisation des assemblées locales dans la formation du Royaume de Norvège. Entre le $\mathrm{x}^{\mathrm{e}}$ et le XIII ${ }^{\mathrm{e}}$ siècle, les habitants, autour du Sognefjord, ont organisé des assemblées impliquant des régions de plus en plus nombreuses. Autour de Gulen, situé au sud du Sognefjord, deux courtyard sites, encore actifs au $\mathrm{x}^{\mathrm{e}}$ siècle, sont connus et un autre, Hjelle fut abandonné dès 880 (un quatrième, $B ø$, pourrait être évoqué, mais sa configuration est assez éloignée des autres courtyard sites). Celui de Sausjord, dans la région de Voss, était situé à la frontière du district d'Aurland, qui appartenait à la région de Sogn : l'hypothèse de $\mathrm{F}$. Iversen est qu'il pourrait donc s'agir d'un lieu de réunion entre les habitants des deux régions. Ainsi, les hommes venus de douze districts différents se seraient réunis à Sausjord : les douze maisons qui entouraient la cour centrale auraient donc servi, selon cette hypothèse, à abriter les délégations venues de chacun des douze districts. Le site de Gjerland aurait quant à lui servi à accueillir les assemblées entre les habitants des deux régions de Sogn et de Firda ou seulement de deux districts limitrophes. Au cours du X ${ }^{\mathrm{e}}$ s., ces sites frontaliers furent abandonnés au profit de Gulen, à la frontière des trois régions de Sogn, Firda et Hordaland. Un processus d'intégration et de rassemblement était à l'œuvre, sans que l'on puisse dire s'il était décidé par les habitants eux-mêmes, soucieux de régler les conflits entre des régions de plus en plus intégrées, ou par les élites locales. Comme le souligne, O. Vésteinsson, dans son article, les élites islandaises agissaient sans doute comme ces élites norvégiennes : le pouvoir central, qui fut pendant longtemps une idée plus qu'une réalité, pouvait être utilisé comme une stratégie, avec des conséquences régionales immédiates, mais ce recours impliquait aussi, sur le long terme, une intégration des régions norvégiennes au système monarchique tout en maintenant, localement, pendant longtemps, des mécanismes permettant des prises de décision indépendantes.

Comme on peut le constater, ce n'est pas le moindre des mérites de ce volume que de proposer, d'une contribution à l'autre, des échos pertinents : il apparaît ainsi, malgré la diversité des terrains et des sources envisagés, le fruit d'une véritable réflexion commune et non une simple collection d'articles.

Corinne PÉNEAU

EA 4392 - CRHEC / Université Paris-Est

Créteil 